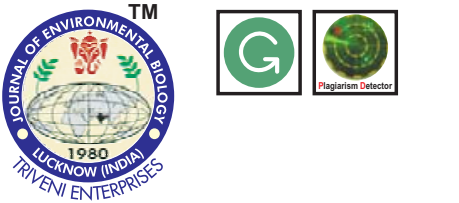

DOI : http://doi.org/10.22438/jeb/38/6/MRN-490

\title{
Characterization of nanorosemary and encapsulated rosemary nanoparticles and their effect on lead induced toxicity in Wistar rats
}

ISSN: 0254-8704 (Print) ISSN: 2394-0379 (Online) CODEN: JEBIDP

\section{JEB Journal of}

Environmental

Biology
Authors Info

P. Virk ${ }^{1 *}$, M. Elobeid', M.A.Awad ${ }^{2}$, A.A. Hendi ${ }^{3}$, K.M.O. Ortashi and M.I. Siddiqui ${ }^{1}$

'Department of Zoology, King Saud University, Riyadh, 11495,

Saudi Arabia

${ }^{2}$ King Abdullah Institute for Nanotechnology, King Saud University, Riyadh, 11495, Saudi Arabia

${ }^{3}$ Department of Physics, King Saud University, Riyadh, 11495, Saudi Arabia

${ }^{4}$ Department of Chemical Engeering,King Saud University, Riyadh, 11495, Saudi Arabia

${ }^{*}$ Corresponding Author Email : virkg@hotmail.com

Key words

Lead

Nanoencapsulation

Oxidative stress

Rosemary leaf extract

Publication Info

Paper received: 19.10.2016

Revised received: 11.02.2017

Re-revised received : 25.04 .2017

Accepted: 17.05.2017

\section{Abstract}

Aim : Lead-induced toxicity is responsible for physiological disarray in humans and animals. The present study was aimed to synthesize'green'nanoparticles of rosemary (Rosmarinus officinalis) leaf extract and investigate the comparative ameliorative efficacy of rosemary extract and the nanoformulations against the lead toxicity in male Wistar rats.

Methodology : Non-capsulated rosemary particles were synthesized with dichloromethane. Encapsulated nanoparticles of rosemary were synthesized with two polymers, starch and a combination of starch with polyvinyl alcohol. The nanoparticles were characterized by transmission electron microscopy and dynamic light scattering.Parallel to this, Wistar rats were exposed to $30 \mathrm{mg} \mathrm{Kg}^{-1}$ lead acetate, four experimental groups were treated with rosemary extract $\left(150 \mathrm{mgKg}^{-1}\right)$ as bulk (BR), nanorosemary (RN), encapsulated nanoparticles RN1 and RN2, respectively, for a period of 21 days.

Results : Green synthesis of water soluble nanorosemary (RN) and encapsulated nanoparticles RN1 and RN2, with composite and single polymer, was successfully achieved. The micrographs showed spherical and irregular morphology of RN, while RN1 and RN2 were more spherical.The average size of nanorosemary (RN) was $75.45 \mathrm{~nm}$, while that of encapsulated rosemary nanoparticles (RN1) and RN2 was $2.665 \mathrm{~nm}$ and $3.026 \mathrm{~nm}$. Treatment with nanoformulations reduced the level of serum malondialdehyde in the rats. Also, due to their enhanced bioavailability, the nanoparticles efficiently reduced the lead concentrationin tissues compared to the bulk rosemary.

Interpretation : The results demonstrate the enhanced protective efficacy of encapsulated nanoparticles, in particular RN1. Thus, the formulation could be a potential ecofriendly, safe and novel therapeutic approach against lead toxicity.

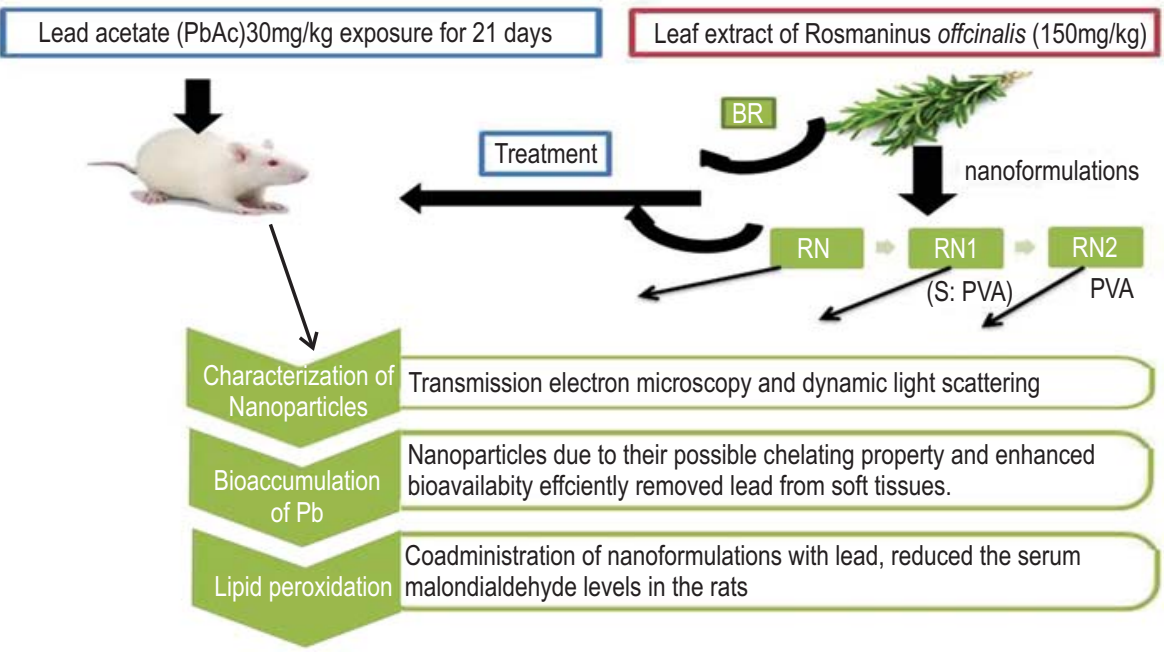




\section{Introduction}

Lead is a potent heavy element ubiquitous in the environment. Although lead toxicity has been extensively researched and reported, it remains a widespread occupational and environmental problem throughout the world. Owing to its unique physical and chemical properties, it is extensively used in industrial processes. It is well documented that $\mathrm{Pb}$ can cause neurotoxicity, nephrotoxicity and deleterious effects on the hematological and cardiovascular systems (ATSDR,2007). Lead exposure occurs mainly through the respiratory and gastrointestinal systems. Lead-induced hepatic damage is primarily caused by oxidative stress, a disturbance of the prooxidant-antioxidant balance by generation of reactive oxygen species manifested as lipid per oxidation (Gurer and Ercal, 2000; Flora et al., 2012). Although chelation therapy is preferred for metal intoxication , the associated side effects of chelating agents has favored the use of natural antioxidants for alleviating metal induced oxidative tissue damage (Flora and Pachauri, 2010). Plants, including herbs and spices, are endowed with phytochemicals which are potential source of natural antioxidants, e.g. phenolic diterpenes, flavonoids, tannins and phenolic acids (Dawidowicz et al., 2006; Erkan et al., 2008). Rosemary (Rosmarinus officinalis) is a native to the Mediterranean and is widely used in cosmetics as culinary herb.It is commonly used as a spice and flavoring agent in food processing (Saito et al., 2004; Panda, 2009; Ibarra et al., 2010). Rosemary leaf extract has also been proposed by the European Community Reference Laboratory as a feed additive (Dossier no. FAD -2004-0003) in the classof antioxidants. The most active antioxidative constituents of rosemary are phenolic diterpenes (carnosic, carnosol, rosmanol, rosmadial, 12-methoxycarnosic acid, epi-, and iso-rosmanol) and phenolic acids (rosmarinic and caffeic) (Brewer, 2011). Carnosic acid has several times the antioxidative activity as BHT and BHA (Richheimer et al.,1996). Rosemary leaf extract in particular, has been demonstrated to possess strong antioxidant activity, predominantly due to the carnosic acid which is present in the alcoholic extract $(10 \%)$. This effectiveness of rosemary extract as an antioxidant has led to its commercial exploitation. The major drawback in using the herbal antioxidants as nutratherpeutics is their low bioavailability. However, the efficacy of such natural antioxidants can be improved by enhancing their bioavailability. Thus, current strategies in targeted drug delivery can be an alternative and promising approach in administration of these compounds. These approaches include improved formulations for better delivery such as liposomes, micelles, phospholipid complexes and nanoparticles (Anand et al., 2007). Further, nano formulations of the antioxidants offer enhanced drug delivery system and has proved to be more effective(Yadav etal., 2012; Flora et al., 2012).

Green synthesis of nanoparticles and nano encapsulation is a novel approach to enhance the bioavailability of phytocompounds. Starch is a natural biopolymer and provides a stable nanocomposite along with polyvinyl alcohol. The present study is an attempt to synthesize and characterize nanorosemary and nano encapsulated rosemary using starch and polyvinyl alcohol by a simple and cost effective method. The study alsocompares the efficacy of bulk rosemary leaf extract to nanorosemary and nano encapsulated rosemary incombating lead-induced oxidative stress and reversing the bioaccumulation of metal in rat model.

\section{Materials and Methods}

Synthesis of Nanorosemary (RN) : Rosemary nanoparticles were prepared from $150 \mathrm{mg}$ of rosemary leaf extract powder $(10 \%$ carnosic acid, Hunan Geneham Biomedical Technology Ltd. China) dissolved in $25 \mathrm{ml}$ dichloromethane (99.5-99.8\%; Merck, Singapore) and mixed with a magnetic stirrer. Further, $3 \mathrm{ml}$ rosemary solution was sprayed into $60 \mathrm{ml}$ boiling water dropwise at a flow rate of $0.2 \mathrm{ml} \mathrm{min}-1$ in 5 min under ultrasonic conditions (ultrasonic power of $100 \mathrm{~W}$ and a frequency of $30 \mathrm{kHz}$ ). After sonication for $20 \mathrm{~min}$, the contents were stirred at 200-800 rpm at room temperature for about $30 \mathrm{~min}$ and then dried.

Synthesis of Rosemary nanoparticles encapsulated in (S and PVA) composite polymers (RN1) : Nanoparticles encapsulated in composite polymers were prepared with rosemary extract: starch: polyvinyl acohol (1:5:5 w/w/w) by nano precipitation technique (Bilati et al., 2005; Zili et al., 2005). Rosemary powder $(150 \mathrm{mg})$ and appropriate amount of starch $(20$ $\mathrm{wt} \%$ of helical amylase, $80 \mathrm{wt} \%$ of branched amylopectin, and 16 wt $\%$ of moisture,Aldrich, Germany) were dissolved in $90 \mathrm{ml}$ of ethanol. The internal organic phase solutions were quickly injected into $200 \mathrm{ml}$ external aqueous solution containing appropriate amount of polyvinyl alcohol (BDH Chemicals, England) and the solutions were then homogenized at $35,000 \mathrm{rpm}$ for $35 \mathrm{~min}$. Ethanol was completely removed and freeze-dried. The nanoparticles powder were collected and stored until further use.

Synthesis of Rosemary nanoparticles encapsulated in PVA polymer (RN2) : The rosemary nanoparticles encapsulated in PVA polymer was prepared with rosemary extract: polyvinyl alcohol (1:10: w/w/w) by the nano precipitation technique (Bilati et al., 2005; Zili et al., 2005). Rosemary powder (150mg) and half amount of PVA were dissolved in $90 \mathrm{ml}$ of ethanol. The internal organic phase solutions were quickly injected into the $200 \mathrm{ml}$ external aqueous solution containing the residual amount of PVA, and then the solutions were homogenized at $35,000 \mathrm{rpm}$ for $35 \mathrm{~min}$. The ethanol was completely removed, and then freeze-dried. The nanoparticles powder were collected and stored until further use.

Experimental design : Forty two male Wistar rats (150-200 g) were procured from the Animal House Facility at King Saud University, Riyadh in the month of April, 2015. The study protocol was approved by the Institutional Animal Ethics Committee (IAEC) of Zoology Department, College of Science, King Saud University. The animals were acclimatized to laboratory conditions for two weeks prior to the experimental period. The animals were divided into six groups each comprising seven rats. Group I was considered as negative control and the rats were 
administered only normal saline. Rats of group II were to treated with $30 \mathrm{mg} \mathrm{kg}^{-1}$ lead acetate. Rats of group III were treated with 30 $\mathrm{mg} \mathrm{kg}^{-1}$ lead acetate and $150 \mathrm{mg} \mathrm{kg}^{-1}$ of rosemary leaf extract. Rats of group IV were adminestrened $30 \mathrm{mg} \mathrm{kg}^{-1}$ and lead acetate with $150 \mathrm{mg} \mathrm{kg}^{-1}$ nanorosemary. Rats of group $\mathrm{V}$ were given $30 \mathrm{mg} \mathrm{Kg}^{-1}$ lead acetate with $150 \mathrm{mg} \mathrm{kg}^{-1}$ nano encapsulated rosemary (RN1). Rats of group VI were given $\mathrm{mg} \mathrm{kg}^{-1}$ lead acetate 30 with $150 \mathrm{mg}$ $\mathrm{kg}^{-1}$ nano encapsulated rosemary (RN2).

The doses were given daily at 8:00 am. During the experimental period, the rats were fed with laboratory chow. Blood was drawn by the orbital sinus venipuncture from the rats of each experimental group and serum was prepared. The serum was prepared by drawing the blood into red stoppered tubes and left at room temperature for clotting followed by centrifugation at $3500 \mathrm{rpm}$ for $15-20 \mathrm{~min}$. The animals were sacrificed and dissected to excise the liver, kidney and spleen. The tissues and serum were storedat $-80^{\circ}$ Cuntil further analysis. The serum was used for analysis of MDAlevel.

Characterization of nanoparticles : The average particle size of nanoparticles of rosemary were measured by dynamic light scattering performed on Zetasizer (Nano series, HT Laser, ZEN3600 from Molvern Instrument, UK). The intensity of scattered light was detected at $90^{\circ}$ to the incident beam. Aqueous solutions of nanoparticles of rosemary (RN, RN1, RN2) were placed in a quartz cuvettes for estumation. Transmission electron microscopy (TEM) was performed (JEM-1011, JEOL, Japan) to assess the morphology of prepared nanoparticles. The samples were prepared by placing a drop of aqueous dispersion of nano formulations on the copper grid and allowing it to air dry.

Determination of lead in tissue : Tissue samples $(0.5 \mathrm{~g}$ each) were digested in a Teflon vessel with $3 \mathrm{ml}$ concentrated $\mathrm{HNO}_{3}$ and kept overnight at $85^{\circ} \mathrm{C}$. Thereafter, $1 \mathrm{ml} 30 \% \mathrm{H}_{2} \mathrm{O}_{2}$ was added and the mixture was heated to $85^{\circ} \mathrm{C}$ for $1 \mathrm{hr}$. The clear supernatant was diluted to $10 \mathrm{ml}$ with deionized water. An aliquout of $5 \mu \mathrm{l}$ was mixed with $5 \mu \mathrm{l}$ modifier (EDTA). The lead concentration in the sample was determined with Atomic Absorption Spectrometer (Model AA240 Z, Agilent Technologies, Santa Clara, USA) at $217 \mathrm{~nm}$ wavelength and detection limit of $4.4 \mu \mathrm{gl}^{-1}$. The lead concentration in the tissues was expressed as $\mu \mathrm{g} \mathrm{g}^{-1}$ wetwt.

Determination of serum MDA level : MDA level in serum was determined with the Alliance Waters High performance Liquid Chromatography (HPLC) 2695 system and a multi fluorescence detector, Model 2475. This system was operated by a Dell Optiplex GX1 computer and Empower software. The reversedphase analytical HPLC column was a ODS Hypersil from Thermo Scientific $(4.6 \mathrm{~mm} \times 25.0 \mathrm{~cm} \times 5 \mu \mathrm{m})$. A guard column, Waters Symmetry TM C18 (4.6 $\mathrm{mm} \times 2 \mathrm{~cm}, 5-\mu \mathrm{m}$ particle size) with the same packing materials was placed in front of the analytical column for protection. Elution was carried out at a flow rate of 1.0 $\mathrm{ml} \mathrm{min}{ }^{-1}$. The column effluent was quantified at an excitation and emission wavelengths of 515 and $553 \mathrm{~nm}$ respectively. Run time per sample was 4.0 min. Aliquots of $25 \mu \mathrm{l}$ of serum were mixed thoroughly with $1 \mathrm{ml}$ of TBA reagent and $10 \mu \mathrm{l}$ of $5 \%$ BHT solutions, vortexed for $2 \mathrm{~min}$ and then heated it for $60 \mathrm{~min}$ in a water bath at $95^{\circ} \mathrm{C}$. Thereafter, the sample was cooled for $20 \mathrm{~min}$ and then centrifuged at $4,000 \mathrm{rpm}$ for $15 \mathrm{~min}$. Supernatants were transferred to a glass vial, and a $5-\mu$ laliquot was injected into column. The stock solution of TEP $(10 \mu \mathrm{mol} \mathrm{ml}-1)$ was prepared by dissolving $240 \mu \mathrm{l}$ of $4.176 \mathrm{M}$ TEP in $100 \mathrm{ml}$ ethanol and stored at $4^{\circ} \mathrm{C}$. The intermediate working standards were prepared by diluting the MDA stock solution with water to concentrations of $0.5,1.0,2.5,5.0,7.5$ and $15 \mathrm{nmol} \mathrm{ml}^{-1}$. The MDA level in the samples was expressed as nmol ml ${ }^{-1}$ of serum.

Statistical analysis : All the data presented expressed as mean values \pm SE. One-way analysis of variance (ANOVA) was performed followed by unpaired student's t-test to analyze group differences. Numerical data was analyzed using SPSS correlated with SPSS 16.0 statistical software (Chicago, IL, USA). The significance level was set to $p \leq 0.05$.

\section{Results and Discussion}

Chelation therapy is the most accepted and recommended strategy to combat metal toxicity, including lead poisoning. However, chelation therapy is always coupled with drawbacks such as toxic effects of the chemicals, low specificity and poor bioavailability of lipophilic compounds (Flora et al., 2013). Natural antioxidants in the form of phytocompounds offer a more safer and novel approach and can be used as chelates against metal toxicity. An increased bioavailability of phytocompounds is be possible through nanosizing these compounds. Novel methods of phytosynthesis of nanoparticles has attracted considerable attention lately owing to their biocompatibility, low toxicity, cost-effectiveness and ecofriendly nature (Rajagopal et al., 2015). Nano formulations attempted in the present study emphasize on the 'green synthesis' of nanoparticles which is ecofriendly and safe. The nanorosemary are natural nanoparticles as they have no chemical stabilizers and metals. Eencapsulation also makes use of a natural polymer, starch along with PVA. The synthesis of nanoparticles of rosemary, as nanorosemary (RN) and encapsulated nanoparticles (RN1 and RN2), were confirmed by DLS and TEM images. The average size of nanorosemary (RN) was $75.45 \mathrm{~nm}$ (Pdi 0.244), rosemary nanoparticles encapsulated with starch and polyvinye alcohol composite polymers (RN1) was $2.665 \mathrm{~nm}$ (Pdi 0.113) and rosemary nanoparticles encapsulated with PVA polymer (RN2) was $3.026 \mathrm{~nm}$ (Pdi 0.084) respectively. All nanoparticles, RN, RN1 and RN2 showed mono-dispersity (Fig. 1A, B and C) which can be observed clearly from a single peak in the DLS graphs. The TEM images of nanoparticles (RN, RN1 and RN2) in Fig.2 also demonstrated mono dispersity of the particles, which corresponds to the DLS results. The electron micrographs showed spherical and irregular morphologies of RN, while RN1 and RN2 represented a more spherical morphology as these particles were encapsulated by polymers. Encapsulation with 

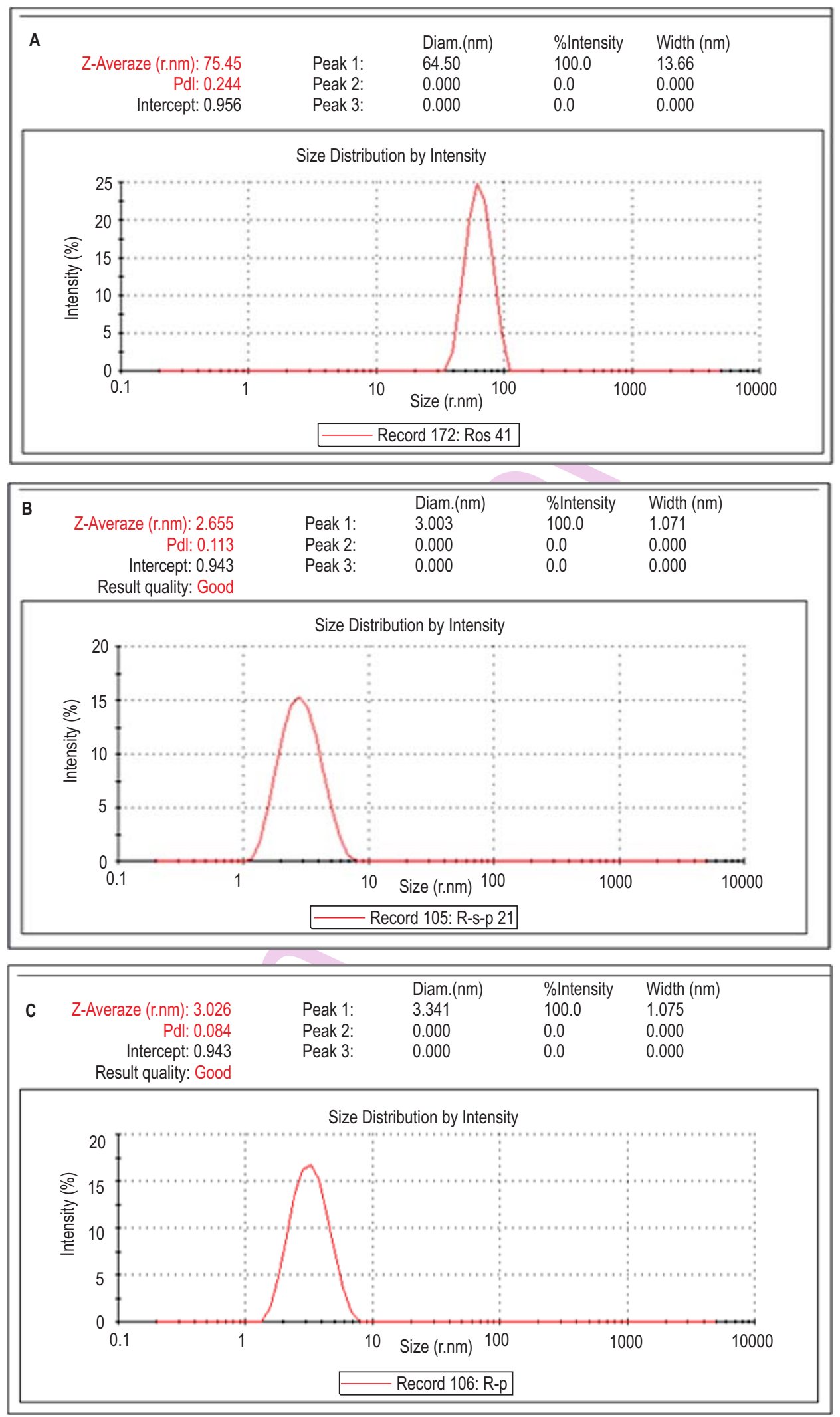

Fig. 1 : Nanoparticle size characterization using dynamic laser light scattering; (A) Rosemary nanoparticles (RN); (B) Rosemary nanoparticles encapsulated in starch and PVA polymers (RN1) and (C) Rosemary nanoparticles encapsulated in PVA polymer (RN2) 

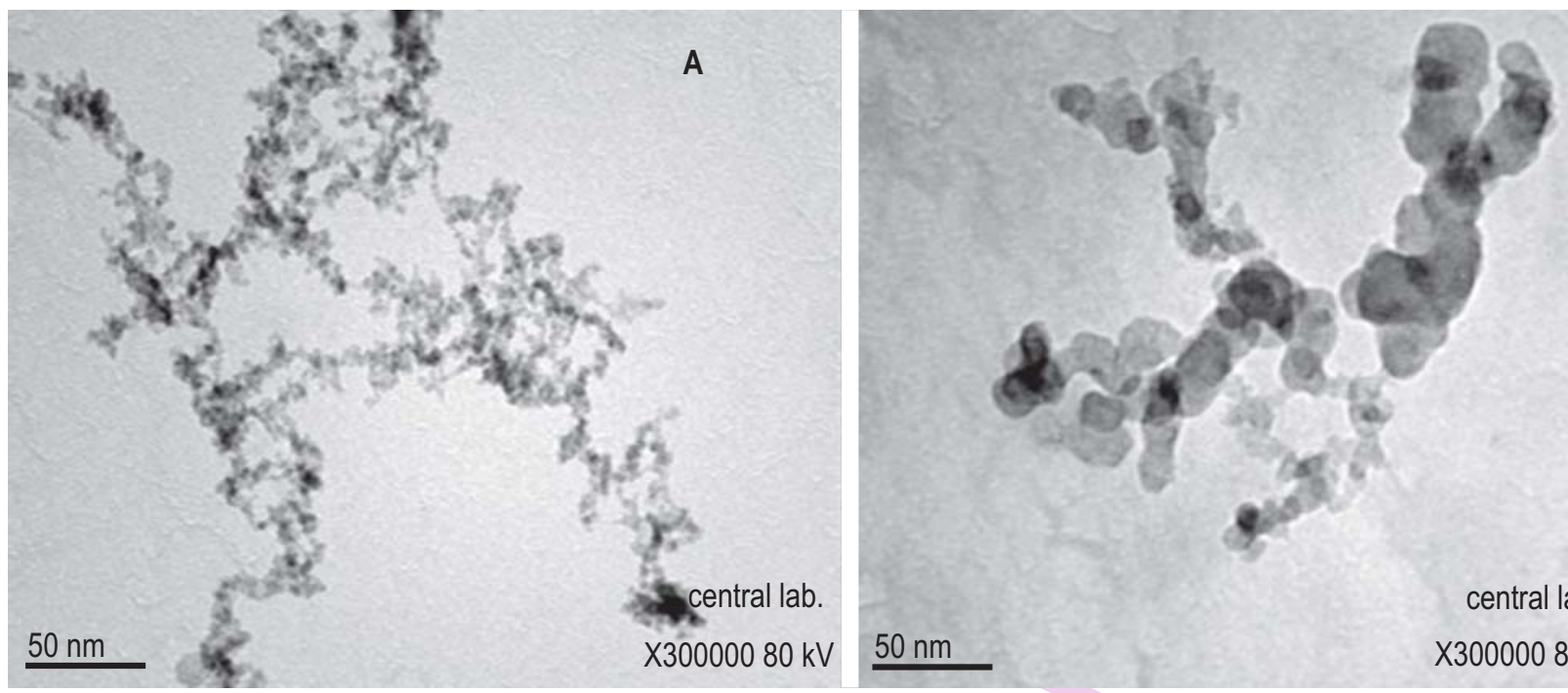

central lab.

X300000 80 kV

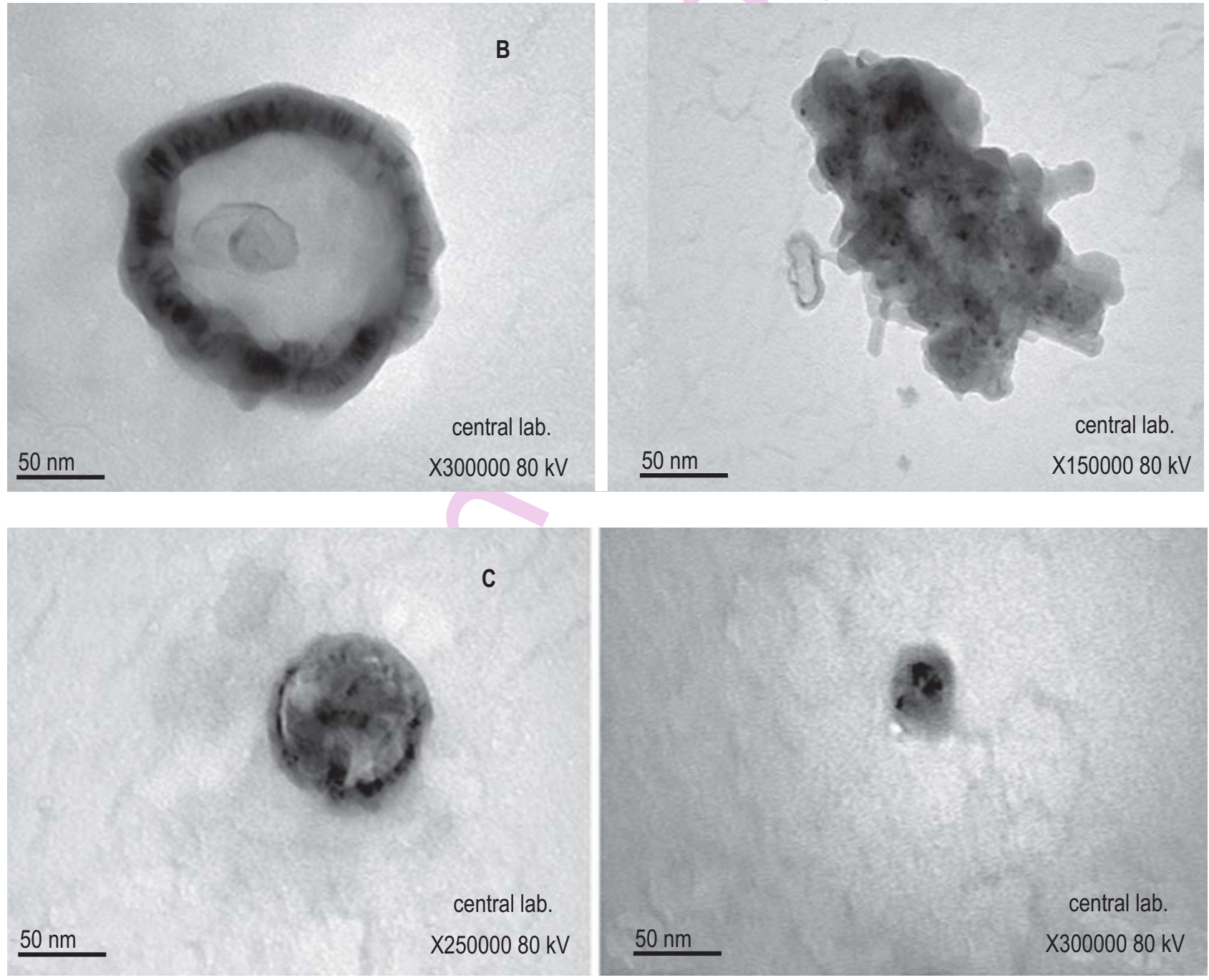

Fig. 2 : Electron micrographs showing morphology of the nanoformulations: (A) Rosemary nanoparticles (RN); (B) Rosemary nanoparticles encapsulated in starch and PVA polymers(RN1); (C) Rosemary nanoparticles encapsulated in PVA polymer(RN2) 
Table 1 : Lead concentrations $\left(\mu \mathrm{g} \mathrm{g}^{-1}\right)$ in brain, liver, kidneys and muscle of rats from different experimental groups

\begin{tabular}{lllll}
\hline Experimental groups & Brain & Liver & Kidneys & Muscle \\
\hline Grp I & $0.0086 \pm 0.00529^{\mathrm{a}}$ & $0.0362 \pm 0.00174^{\mathrm{a}}$ & $0.0020 \pm 0.00200^{\mathrm{a}}$ & $0.0812 \pm 0.00338^{\mathrm{a}}$ \\
Grp II & $0.4300 \pm 0.001761^{\mathrm{b}}$ & $1.6040 \pm 0.02182^{\mathrm{b}}$ & $3.2760 \pm 0.07679^{\mathrm{b}}$ & $0.2820 \pm 0.01393^{\mathrm{b}}$ \\
Grp III & $0.2100 \pm 0.01304^{\mathrm{c}}$ & $1.6400 \pm 0.07483^{\mathrm{b}}$ & $2.4860 \pm 0.02993^{\mathrm{c}}$ & $0.1260 \pm 0.00678^{\mathrm{c}}$ \\
Grp IV & $0.1400 \pm 0.01140^{\mathrm{d}}$ & $1.0620 \pm 0.01241^{\mathrm{c}}$ & $3.0880 \pm 0.01158^{\mathrm{b}}$ & $0.1380 \pm 0.00374^{\mathrm{c}}$ \\
Grp V & $0.0520 \pm 0.00374^{\mathrm{a}}$ & $1.3460 \pm 0.00748^{\mathrm{d}}$ & $2.5400 \pm 0.09274^{\mathrm{c}}$ & $0.0880 \pm 0.00663^{\mathrm{a}}$ \\
Grp VI & $0.2280 \pm 0.01020^{\mathrm{c}}$ & $1.3340 \pm 0.01364^{\mathrm{d}}$ & $2.5380 \pm 0.02010^{\mathrm{c}}$ & $0.1380 \pm 0.00374^{\mathrm{c}}$ \\
\hline
\end{tabular}

Values with different superscript letter within each column are significantly different at $5 \%$ probability level. Values are mean of five replicates $\pm \mathrm{SE}$

composite polymer produced a better nanoparticle in terms of nanoscale that enhanced it efficacy as a therapeutic agent.

The results of the biological assays demonstrate that nano formulation retained the antioxidant and metal-chelating properties of rosemary leaf extract. All the nano formulations formed were physically and chemically stable with monodispersity, readily dispersible in water, and could be stored at room temperature over a period of time without any decomposition or aggregation. The enhanced aqueous solubility of nanoparticles could be attributed to their larger surface area, which promotes dissolution ( McNeil, 2005).

A significant $(p \leq 0.05)$ increase was observed in the $\mathrm{Pb}$ concentration in tissues after an exposure period of 21 days. Heavy metals such as lead have been reported to accumulate in the tissues of vertebrates from diverse taxa and environments (Mehrotra et al., 2008).Treatment with bulk rosemary extract and its nano formulations (RN, RN1, RN2) significantly reduced lead bioaccumulation in the tissues. Lead concentration in the brain of the group II rats $\left(0.4300 \mu g^{-1}\right)$ was significantly $(p \leq 0.05)$ higher in comparison to that of group I rats $\left(0.0086 \mu \mathrm{g} \mathrm{g}^{-1}\right)$. In comparison to group II rats, all lead exposed rats treated with rosemary and their nanoparticles ( $B R, R N, R N 1, R N 2$ ) showed a significant ( $p \leq 0.05$ ) reduction in the lead concentration, being most effective in group $V$ treated with RN1. $\left(0.0520 \mu \mathrm{g} \mathrm{g}^{-1}\right)$. Within the treated groups, lead concentration in group III rats $\left(0.210 \mu \mathrm{g} \mathrm{g}^{-1}\right)$ was significantly $(p \leq$ 0.05 ) higher than groups $V$ and $V I$ rats but was comparable to the group IV rats (Table 1). There was also a significant $(p \leq 0.05)$ increase observed in the lead concentration in the kidneys from group II (3.2760 $\left.\mu \mathrm{g} \mathrm{g}^{-1}\right)$ in comparison to group II $\left(0.0020 \mu \mathrm{g} \mathrm{g}^{-1}\right)$. Treatment with rosemary and its encapsulated nano formulations significantly ( $p \leq 0.05)$ reduce the lead burden in the kidneys. However, no significant effect on the lead concentration was observed in group IV rats. The effect of RN1 and RN2 was to comparable eachother (Table 1). Further, the lead concentration in the liver of rats exposed to lead only $\left(1.6040 \mathrm{\mu g} \mathrm{g} \mathrm{g}^{-1}\right)$ showed a significant $(p \leq 0.05)$ increase when compared to the control group $\left(0.0362 \mu \mathrm{g} \mathrm{g}^{-1}\right)$. Treatment with bulk rosemary (BR) did not have a significant effect on the lead concentration. Howeve, treatment with nanoparticles of rosemary ( NR, NR1, NR2) significantly ( $p \leq$
Table 2 : Serum MDAlevels $\left(\mathrm{nmol} \mathrm{ml^{-1 }}\right.$ ) of rats from different experimental groups

\begin{tabular}{ll}
\hline Experimental groups & Serum MDA levels \\
\hline Grp I & $2.0942 \pm 0.00684^{\mathrm{a}}$ \\
Grp II & $3.5302 \pm 0.6596^{\mathrm{b}}$ \\
Grp III & $2.3816 \pm 0.02561^{\mathrm{C}}$ \\
Grp IV & $2.5512 \pm 0.02560^{\mathrm{d}}$ \\
Grp V & $1.6869 \pm 0.00400^{\circ}$ \\
Grp VI & $1.6215 \pm 0.04687^{\mathrm{e}}$ \\
\hline
\end{tabular}

Values with different superscript letter within each column are significantly different at $5 \%$ probability level. Values are mean of five replicates \pm SE

0.05 ) reduced the lead concentration in comparison to lead control group. Treatment with NR was significantly effective in reducing the lead concentration in liver than NR1 and NR2 (Table 1). A significant ( $p \leq 0.05$ ) increase in lead concentration also was observed in the muscles of rats exposed to lead only $(0.2820 \mu \mathrm{g} \mathrm{g}$ $\left.{ }^{1}\right)$ in comparison to control group $\left(0.0812 \mu \mathrm{g} \mathrm{g}^{-1}\right)$. Treatment with rosemary leaf extract, both as bulk and nano formulations significantly ( $p \leq 0.05$ ) reduced the lead concentration in comparison to group II rats. Among the treated groups, group V $\left(0.0880 \mu \mathrm{g} \mathrm{g}^{-1}\right)$ rats showed least lead concentration in the muscles and was comparable to the control (Table 1).

Thus, the results on the bioaccumulation of lead in the present study showed a marked ameliorative effect of rosemary leaf extract, which was evident from a reduction in bioaccumulation of metal in target tissues. Futhermore, an assessment of lipid peroxidation showed that the serum MDA levels were significantly $(p \leq 0.05)$ elevated in group II rats, $\left(3.5302 \mathrm{nmolm}^{-1}\right)$ in comparison to the control group (2.0942 nmol $\left.\mathrm{ml}^{-1}\right)$. Treatment with rosemary, both as bulk (BR) and nanoparticle ( $R N, R N 1, R N 2)$ significantly $(p \leq 0.05)$ reduced the serum MDA levels. However, within the treated groups ? treatment with encapsulated rosemary nanoparticles (RN1 And RN2) was more effective in reversing the effect of lead on lipid peroxidation (Table 2), this could be attributed to the antioxidant activity of rosemary extract primarily due to the presence of carnosic acid and carnosol (Kadri et al., 2011; Machado et al., 
2013). Similar findings on the ameliorative potential of rosemary leaf extract against metal toxicity and associated oxidative stress has previously been reported (Virk et al., 2013; Sakr et al., 2015; Al-Anazi et al., 2015; Rašković et al.,2015). The results on lead bioaccumulation in tissues clearly showed the mitigating effect of rosemary extract, both as bulk and nanoparticles. An overall assessment of the results showed that nano formulations of rosemary were more effective in chelating the metal from the tissues in comparison to the bulk extract used. This is in consensus with a similar experimental study on rat model by Flora et al. (2013) where nanocurcumin due to its possible chelating property and enhanced bioavailability efficiently removed lead from blood and soft tissues compared to bulk curcumin. Within three different types of nano formulations used in the present study, the encapsulated nanoparticles (NR1 and NR2) were more effective in comparison to nanorosemary, owing to the increased stability and bioavailability due to encapsulation. Further, the encapsulated nanoparticles with composite polymer was most effective in enhancing the chelating activity of rosemary extract, which could be attributed to the fact that encapsulation efficiency was enhanced with a composite polymer, a combination of starch and PVA, as suggested in earlier studies on nano encapsulation with composite polymers (Sehra et al., 2005; Mu and Zhong, 2006). The lead -induced oxidative stress was evident by an enhanced level of lipid peroxidation in exposed rats. Treatment with rosemary, bulk and nanoparticles markedly reduced the serum MDA levels and were effective in combating the oxidative stress. The antioxidative effect of rosemary leaf extract against metal induced oxidative stress has been previously reported (Virk et al., 2013; Al-Anazi et al., 2015; Sakr et al., 2015). This could be attributed to the presence of a major a constituent, carnosic acid in the leaf extract used in the present study. The nanoformulation proved to be more effective in reducing the lipid peroxidation, and the effect was more profound in encapsulated nanoparticles.

Thus, a broad assessment of the biological end points evaluated to investigate the therapeutic efficacy of rosemary and its nano formulations showed that the nanoparticles enhanced the protective effect of rosemary leaf extract against lead-induced toxicity in rats. Which can be explained on the fact that nanosizing the particles enhances bio availability making the drug /compound more efficacious. In addition, encapsulation of rosemary extract was more effective as an antioxidant and chelator as encapsulation with polymers made the nanoparticles more stable, and further enhanced their bioavailability. This was evident from the smaller size of encapsulated nanoparticles in comparison to nanorosemary. The success of application of nanoparticles as therapeutic agents depends on their ability to evade the reticulo endothelial system that ensures a prolonged circulation time in the blood (Guo and Huang, 2011). The results of the study show that polymer encapsulated nanoparticles have distinct properties, which aided in evading the RES uptake, and hence had a longer circulation time in blood and provided a sustained release of rosemary extract. Similar results were reported in a study on arsenic-induced toxicity in rats, where curcumin encapsulated in chitosan nanoparticles was more effective than free cucurmin (Yadav et al., 2012).

The key findings of the study suggest that rosemary nanoparticles with encapsulation can be an effective nutritional intervention against lead toxicity, as rosemary leaf extract is an established dietary antioxidant. This alternative drug delivery approach would possibly bring out rosemary leaf extract as an effective and promising agent to treat metal-induced toxicity.

\section{Acknowledgment}

This research project was supported by a grant from the "Research Center of the Female Scientific and Medical Colleges", Deanship of Scientific Research, King SaudUniversity, Riyadh, SaudiArabia.

\section{References}

Agency for Toxic Substances and Disease Registry (ATSDR): Toxicological profile for Lead. Atlanta, GA: U.S. Department of Health and Human Services, Public Health Service (2007).

Al-Anazi, M.S., P. Virk, M. Elobeid and M.I. Siddiqui: Ameliorative effects of Rosmarinus officinalis leaf extract and Vitamin C on cadmiuminduced oxidative stress in Nile tilapia, Oreochromis niloticus. J. Environ. Biol., $36,1401-8(2015)$.

Anand, P., A.B. Kunnumakkara., R.A.Newman and B.B.Aggarwal : Bioavailability of curcumin: Problems and promises. Mol. Pharm., 4, 807-818 (2007).

Bilati, U., E. Allemann and E. Doelker: Development of a nanoprecipitation method intended for the entrapment of hydrophilic drugs into nanoparticles. Eur. J. Pharm. Sci., 24, 67-75 (2005).

Brewer,M.S.: Natural antioxidants: Sources, compounds, mechanisms of action and potential applications. Compr. Rev. Food Sci. Food Saf.,10, 221-247(2011).

Dawidowicz, A.L., D.Wianowska and B. Baraniak: The antioxidant properties of alcoholic extracts from Sambucus nigra $L$. (antioxidant properties of extracts). LWT Food Sci. technol., 39, 308-315(2006).

Erkan, N., G. Ayranci and E. Ayranci: Antioxidant activities of rosemary (Rosmarinus Officinalis L.) extract, blackseed (Nigella sativa L.) essential oil, carnosicacid, rosmarinic acid and sesamol. Food Chem.,110, 76-82(2008)

Flora, G., D. Gupta and A. Tiwari:Toxicity of lead : A review with recent updates. Interdiscip. Toxicol.,5, 47-58 (2012).

Flora, S.J.S.and V. Pachauri : Chelation in metal intoxication. Int. J. Environ. Res. Public Hlth., 7, 2745-2788(2010).

Flora, G., D. Gupta and A.Tiwari : Nanocurcumin : A promising therapeutic advancement over native curcumin. Crit. Rev.Ther. Drug Carr.Syst., 30,331-68(2013).

Guo, S. and L. Huang : Nanoparticles escaping RES and endosome: Challenges for siRNA delivery for cancer therapy. J. Nanomater., 2011, Article ID 742895, pp 12 (2011).

Gurer, H. and N. Ercal : Can antioxidants be beneficial in the treatment of lead poisoning? Free Radic. Biol. Med., 29, 927-945(2000).

Ibarra, A., J.Cases, A.Bily, K. He, N. Bai, M. Roller ,A. Coussaert and C. Ripoll : Importance of extract standardization and in vitrolex vivo assay selection for theevaluation of antioxidant activity of botanicals : A case study on three Rosmarinus officinalis $L$. 
extracts. J. Med.Food.,13, 1167-1175 (2010).

Kadri, A., Z. Zarai, I. Ben Chobba, A. Bekir, N. Gharsallah, M. Damak and R. Gdoura: Chemical constituents and antioxidant properties of Rosmarinus officinalis L. essential oil cultivated from SouthWestern Tunisia. J. Med. Plants Res., 5,5999-6004 (2011).

Machado, D.G., M.P. Cunha, V.B. Neis, G.O. Balen, A. Colla, L.E. Bettio, A. Oliveira, F.L. Pazini, J.B.Dalmarco, E.L.Simionatto, M.G. Pizzolatti and A.L.Rodrigues: Antidepressant-like effects of fractions, essential oil, carnosol and betulinic acid isolated from Rosmarinus officinalis L. Food Chem.,136,999-1005(2013).

McNeil, S. E. : Nanotechnology for the biologist. J. Leukocyte Biol.,78, 585-592(2005).

Mehrotra,V., V.L. Saxena and A.K. Saxena : Impact of different doses of lead on internal organs of quails. J. Environ. Biol., 29, 147-149 (2008).

$\mathrm{Mu}, \mathrm{X}$. and Z. Zhong: Preparation and properties of poly(vinyl alcohol)stabilized liposomes. Int. J. Pharm., 318, 55-61(2006).11

Panda, $\mathrm{H}$. : Cultivation of Rosmarinus officinalis in aromatic plants cultivation, processing and uses, National Institute of Industrial Research, editor. New Delhi : Asia Pacific Business Press, Inc., pp. 22-28 (2009).

Rajagopal, T., I.A.A., Jemimah, P., Ponmanickam and M. Ayyanar. Synthesis of silver nanoparticles using Catharanthus roseus root extract and its larvicidal effects. J. Environ. Biol., 36, 1283-1289 (2015).

Rašković, A., I. Milanović, N. Pavlović, T. Ćebović, S.Vukmirović and M. Mikov: Antioxidant activity of rosemary (Rosmarinus officinalis L.) essential oil and its hepatoprotective potential. BMC Complement.
Altern. Med.,14, 225 (2014).

Richheimer, S.L., M.W.Bernart, G.A. King, M.C.Kent and D.T. Beiley : Antioxidant activity of lipid-soluble phenolic diterpenes from rosemary. J.Amer. Oil Chem. Soc., 73, 507-514 (1996).

Sakr, S.A., M.F. Bayomy and A.M. El-Morsy :Rosemary extract ameliorates cadmium-induced histological changes and oxidative damage in the liver of albino rat. J. Basic App. Zool., 71, 1-9 (2015).

Saito, Y., A. Shiga, Y. Yoshida, T. Furuhashi, Y. Fujita and E .Niki: Effects of novel gaseous antioxidative system containing a rosemary extract on the oxidation induced by nitrogen dioxide and ultraviolet radiation. Biosci. Biotechnol. Biochem., 68, 781-786 (2004).

Sehra, S. and A.S. Dhake: Formulation and evaluation of sustained release microspheres of poly-lactide-co-glycolide containing tamoxifen citrate. J. Microencaps., 22, 521-528 (2005).

Virk, P., M.Elobeid, S. Hamad, Z.Korany, M. Al-Amin, M. Daghestani, S. Omer, E.Al Olayan, M. I. Siddiqui and N.M.Mirghani : Ameliorative effects of Embilica officinalis and Rosmarinus officinalis on cadmium-induced oxidative stress in Wistar rats. J. Med. Plants Res., 7, 805-818(2013).

Yadav, A., V. Lomash and M. Samim and S.J.S. Flora : Curcumin encapsulated in chitosan nanoparticles: A novel strategy for the treatment of arsenic toxicity. Chem. Biol. Interact., 199, 49-61 (2012).

Zili, Z., S. Sfar and H. Fessi : Preparation and characterization of polyepsilon-aprolactone nanoparticles containing griseofulvin. Int. J.Pharm., 294, 261-267(2005). 